\title{
Use of Rituximab as an Off-Label Medication in Glomerular Diseases: Clinical Perspective
}

\author{
Ali AISahow ${ }^{a}$ Abdullah Al-Muhaiteeb $^{a}$ Hani Nawar ${ }^{a}$ Bassam AlHelal $^{b}$ \\ Anas AlYousef ${ }^{\mathrm{E}}$ Emad Abdallah ${ }^{\mathrm{b}}$ Ahmad AbuShall ${ }^{\mathrm{c}}$ Sameh Elmekawi ${ }^{\mathrm{a}}$ \\ Basem Meshal $^{a}$ Ahmed AlQallafd ${ }^{d}$ Heba AlRajab ${ }^{c}$

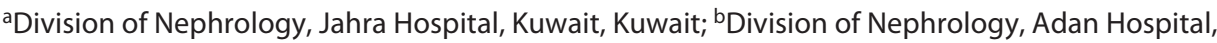

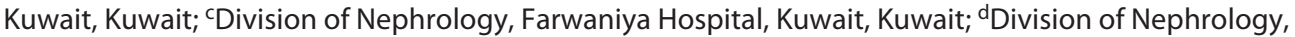

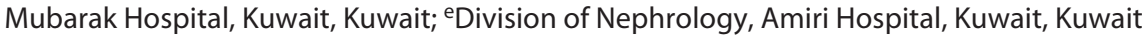

\section{Highlights of the Study}

- Rituximab has been used as an alternative or add-on therapy for glomerulonephritis such as lupus nephritis (LN) and nephrotic syndrome for decades.

- We retrospectively reviewed its use over 4 years in Kuwait for any glomerulopathy and found 61 cases of various glomerulopathies.

- Our data support its use in resistant minimal change disease, membranous nephropathy, LN, and IgG4-related disease.

\section{Keywords}

Rituximab · Lupus nephritis · Minimal change disease · Membranous nephropathy · Kuwait

\begin{abstract}
Objectives: The aim of this study was to review the use rituximab (RTX) and outcomes in immune-mediated glomerular diseases (glomerulonephritis [GN]) and to compare it to the established literature. Methods: Adult GN patients who received RTX between January 2014 and January 2018 in three public hospitals were reviewed. Membranous nephropathy (MN) and minimal change disease (MCD) were considered diseases with the literature supporting RTX use. Lupus nephritis (LN), primary focal segmental glomerulosclerosis $\left(1^{\circ}\right.$
\end{abstract}

karger@karger.com www.karger.com/mpp

Karger $\stackrel{\text { ' }}{5}$
(C) 2022 The Author(s)

Published by S. Karger AG, Basel

This is an Open Access article licensed under the Creative Common Attribution-NonCommercial-4.0 International License (CC BY-NC) (http://www.karger.com/Services/OpenAccessLicense), applicable to the online version of the article only. Usage and distribution for commercial purposes requires written permission.
FSGS), IgA nephropathy, IgG4-related disease (IgG4-RD), and C3GN had insufficient literature support for RTX use. Clinical remission was assessed 6 months after receiving RTX. $\boldsymbol{R e}$ sults: A total of 61 cases were analyzed. RTX was an add-on therapy in $87 \%$. The remission rate was $95 \%$ in the MCD and MN versus $56 \%$ in the off-label group $(p=0.002)$. LN patients had a mean initial estimated glomerular filtration rate (eGFR) of $69 \mathrm{~mL} / \mathrm{min}$. All class III LN achieved remission, and 11 of 21 class IV achieved remission. The mean initial eGFR for $1^{\circ}$ FSGS was $33 \mathrm{~mL} / \mathrm{min}$, and it did not improve, and only 2 of 5 had partial resolution of proteinuria. Proteinuria improved in 3 of 5 IgG4-RD cases with eGFR stabilization but failed to improve in C3GN cases with eGFR deterioration. Vasculitis cases (6 ANCA-associated vasculitis and $2 \lg A$ vasculitis) were analyzed separately. Remission was achieved in only $2 \mathrm{AN}$ -
Correspondence to:

Ali AlSahow, alsahow@ hotmail.com 
CA-associated vasculitis cases, and none in IgA vasculitis cases. Conclusions: Our data support the use of RTX in resistant MCD and MN. RTX showed success in LN and IgG4-RD but not FSGS or C3GN. The small number of cases of vasculitis does not allow drawing a conclusion on RTX effectiveness.

(c) 2022 The Author(s).

Published by S. Karger AG, Basel

\section{Introduction}

Rituximab (RTX) is a chimeric monoclonal antibody targeting the CD20 molecule. Administration of RTX causes the lysis of circulating and tissue-resident $\mathrm{CD} 20^{+}$ cells but not the destruction of stem cells or plasma cells. This results in depletion of memory B cells with decrease in production of antibodies and cytokines, which would alter the functioning of antigen-presenting cells. It is generally well tolerated, and its effects on B-cell depletion can last up to 12 months [1].

The initial Food and Drug Administration (FDA) approval for this medication in 1997 was for non-Hodgkin's lymphoma. RTX has been used as an alternative therapy for glomerulonephritis (GN) for few decades. It has emerged as a vital therapy for immune-mediated glomerular diseases worldwide. These include membranous nephropathy $(\mathrm{MN})$, ANCA-associated vasculitis, minimal change disease (MCD), and primary focal segmental glomerulosclerosis ( $1^{\circ}$ FSGS) [2-6]. However, it has no proven role in IgA nephropathy (IgAN) yet, and its role in lupus nephritis (LN) and C3GN is still not clear [7-10]. Nevertheless, the evidence and indications for RTX use are still evolving and rapidly changing.

Here, we review local RTX use as a salvage therapy, in immune-mediated glomerular disease that failed firstline evidence-based agents, to induce remission despite lack of official indication (i.e., off-label). We wanted to assess the clinical indications of RTX use for glomerular diseases compared to the established literature available and whether remission was achieved, in the hope that our findings may help establish local standards and regulations for RTX use in glomerular diseases.

\section{Methods}

\section{Study Population}

We collected data from patients with glomerular diseases who received RTX in three major tertiary care public hospitals (Jahra Hospital, Farwaniya Hospital, and Adan Hospital) in Kuwait between January 2014 and January 2018. All adult patients aged 18 years and above who received RTX for glomerular disease were included. The decision to administer RTX was solely made by the nephrologist in charge, when first-line therapy failed to achieve remission.

Data Collection, Measurements, and Definitions

Ethical approval was obtained from the Ministry of Health Kuwait University Joint Committee on Medical and Scientific Research. Data collection included age, gender, medications (mainly the use of angiotensin-converting enzyme inhibitor/angiotensin receptor blocker and the use of steroids and other immunosuppressive drugs), immunology tests (if applicable), 24-h urine protein, and estimated glomerular filtration rate (eGFR) estimated by the CKD-EPI formula at time of therapy and at 6 months after treatment.

Patients were separated based on their original glomerular disease. These included MCD, $1^{\circ}$ FSGS, MN, LN, IgAN, IgG4-related disease (IgG4-RD), C3GN, IgA vasculitis, and ANCA-associated vasculitis. All cases included have underwent percutaneous kidney biopsy. $1^{\circ}$ FSGS diagnosis was based on clinical picture in combination with light microscopy findings.

For analysis, MN and MCD were placed in the data supporting RTX use group, based on the available literature. In comparison, $1^{\circ}$ FSGS, LN, IgAN, IgG4-related disease, and C3GN were placed in the group with insufficient data to support RTX use (off-label group).

The primary outcome was clinical remission. This would be either complete or partial remission (PR). Complete remission (CR) was defined as a reduction in proteinuria to $\leq 300 \mathrm{mg} /$ day in standard 24-h urine protein collection [11]. PR was defined as a reduction in proteinuria of $\geq 50$ percent from baseline but more than $300 \mathrm{mg} /$ day and $<3.5 \mathrm{~g} /$ day in the patient presenting with nephrotic range proteinuria [12]. The ANCA-associated and IgA vasculitis were analyzed separately because of their systemic nature and because their remission criteria is different.

\section{Statistical Analysis}

Stata version 16 was used for statistical analysis. Standard descriptive techniques were used to assess and analyze the study population and relevant variables including the indication of RTX. The mean was calculated for continuous variables. Statistical tests comparing categorical variables were done by the $\chi^{2}$ test. Logistic regression model was used to assess the probability of remission if RTX was used as off-label versus an indication supported by the literature.

\section{Results}

\section{Baseline Characteristics}

A total of 75 patients who received RTX for glomerular diseases during the study period were identified (Fig. 1). Three patients were excluded for missing outcome data, and three other patients for refusal of kidney biopsy ( 2 presumed LN and 1 presumed MCD). An additional eight cases of vasculitis (6 cases of pauci-immune ANCA-associated vasculitis and 2 cases of IgA vasculitis) were analyzed separately due to differences in re- 


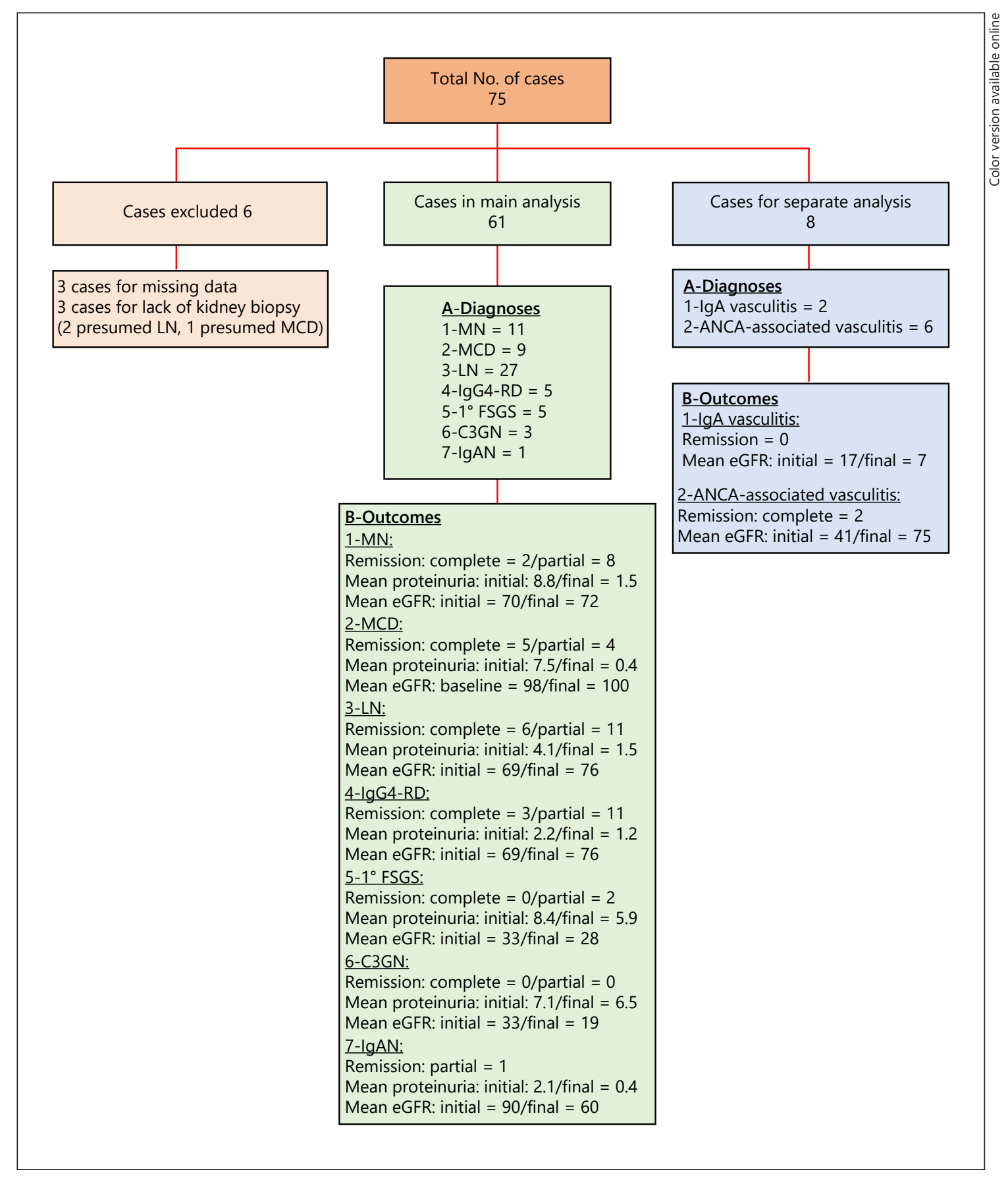

Fig. 1. Flowchart showing the diagnostic distribution of selected cases and their outcomes.

mission criteria and due to their systemic nature. The remaining 61 cases were included in the main analysis. Baseline characteristic of all patients is shown in Table 1. Serum anti-PLA2R antibody testing for $\mathrm{MN}$ was not done but was positive in all of the biopsies. No major side effects were reported. RTX was an add-on therapy (i.e., 1st-line treatment failed) in 55 of the 61 patients and in all of MN and LN cases. RTX was administered mainly using two regimens. These were $375 \mathrm{mg} / \mathrm{m}^{2}$ IV once weekly $\times 4$ doses, which was received by $74 \%$ of the cohort or two doses each of $1 \mathrm{~g}$ infused 2 weeks apart received by $26 \%$ of cases. 
Table 1. Baseline characteristics and treatments received of all patients who received RTX

\begin{tabular}{|c|c|c|c|c|c|c|c|c|c|}
\hline Glomerular diseases & MN & MCD & LN & $\operatorname{lgG} 4-R D$ & $1^{\circ} \mathrm{FSGS}$ & C3GN & $\lg A N$ & $\begin{array}{l}\text { IgA vas- } \\
\text { culitis }\end{array}$ & $\begin{array}{l}\text { ANCA-associat- } \\
\text { ed vasculitis }\end{array}$ \\
\hline Patients, $n(\%)$ & $11(16)$ & $9(13)$ & $27(39)$ & $5(7)$ & $5(7)$ & $3(4)$ & $1(1.5)$ & $2(3)$ & $6(9)$ \\
\hline \multicolumn{10}{|l|}{ Gender } \\
\hline Male & 7 & 6 & 4 & 1 & 3 & 2 & 0 & 2 & 5 \\
\hline Female & 4 & 3 & 23 & 4 & 2 & 1 & 1 & 0 & 1 \\
\hline Mean baseline eGFR, $\mathrm{mL} / \mathrm{min}$ & 70 & 98 & 69 & 57 & 33 & 33 & 90 & 17 & 41 \\
\hline ACE/ARB & 11 & 2 & 17 & 3 & 3 & 2 & 1 & 0 & 2 \\
\hline \multicolumn{10}{|l|}{ Other immune therapies used } \\
\hline Prednisone & 11 & 5 & 25 & 4 & 4 & 3 & 1 & 2 & 5 \\
\hline $\mathrm{CNI}$ & 9 & 1 & 6 & 1 & 3 & 1 & 0 & 0 & 0 \\
\hline MMF & 2 & 3 & 23 & 3 & 4 & 1 & 0 & 2 & 2 \\
\hline \multicolumn{10}{|l|}{ RTX dose } \\
\hline $375 \mathrm{mg} \times 4$ & 7 & 7 & 22 & 3 & 2 & 3 & 1 & 2 & 3 \\
\hline $1 \mathrm{~g} \times 2$ & 4 & 2 & 5 & 2 & 3 & 0 & 0 & 0 & 3 \\
\hline
\end{tabular}

LN, lupus nephritis; MN, membranous nephropathy; MCD, minimal change disease; FSGS, focal segmental glomerulosclerosis; IgAN, IgA nephropathy; GN, glomerulonephritis; UP, urinary protein; eGFR, estimated glomerular filtration rate; ACEI/ARB, angiotensin-converting enzyme inhibitor/angiotensin receptor blocker; CNI, calcineurin inhibitors; MMF, mycophenolate mofetil; RTX, rituximab.

Table 2. Outcomes of all patients

\begin{tabular}{|c|c|c|c|c|c|c|c|c|c|}
\hline Glomerular diseases (cases, $n$ ) & $\mathrm{MN}(11)$ & MCD (9) & LN (27) & $\operatorname{lgG} 4-\mathrm{RD}(5)$ & ) FSGS (5) & C3GN (3) & $\lg A N(1)$ & $\begin{array}{l}\text { IgA vas- } \\
\text { culitis (2) }\end{array}$ & $\begin{array}{l}\text { ANCA-asso- } \\
\text { ciated GN (6) }\end{array}$ \\
\hline $\mathrm{PR}, n(\%)$ & $8(73)$ & $4(45)$ & $11(41)$ & $2(40)$ & $2(40)$ & 0 & 1 & 0 & 0 \\
\hline Total remission, $n(\%)$ & $10(91)$ & $9(100)$ & $17(63)$ & $3(60)$ & $2(40)$ & 0 & 1 & 0 & $2(33)$ \\
\hline Mean final 24 h UP, g/day & 1.5 & 0.4 & 1.5 & 1.2 & 5.9 & 6.5 & 0.4 & - & - \\
\hline Mean final eGFR, mL/min & 72 & 100 & 76 & 60 & 28 & 19 & 60 & 7 & 75 \\
\hline
\end{tabular}

Remission in ANCA-mediated GN and IgA vasculitis: remission was defined as improvement in the eGFR. Remission in others was in improvement in $24 \mathrm{~h}$ urinary protein. MN, membranous nephropathy; MCD, minimal change disease; LN, lupus nephritis; IgG4-RD, IgG4related disorders; FSGS, focal segmental glomerulosclerosis; C3GN, C3 glomerulonephritis; IgAN, IgA nephropathy; UP, urinary protein; eGFR, estimated glomerular filtration rate; S, supported; I, insufficient data.

\section{Clinical Outcome and Remission}

Outcomes in terms of remission of proteinuria and changes in the eGFR are shown in Table 2. The calculated mean initial proteinuria in addition to mean proteinuria at 3 months and at 6 months after RTX administration is depicted in Figure 2, and rates of remissions are shown in Figure 3. The total rates of remission for $\mathrm{MN}$ and $\mathrm{MCD}$ were $91 \%$ and $100 \%$, respectively, while rates of remission for $1^{\circ} \mathrm{FSGS}, \mathrm{LN}$, and IgG4-RD were $40 \%, 63 \%$, and $60 \%$, respectively.

In the off-label group, LN patients had a mean initial eGFR of $69 \mathrm{~mL} / \mathrm{min}$, and it was maintained at 6 months. All five class III LN patients achieved remission with RTX ( 2 complete, 3 partial), and 11 of 21 class IV LN patients achieved remission ( 4 complete, 7 partial). In addition, 1 patient with class $\mathrm{V}$ achieved PR Figure 4 . The mean ini- 


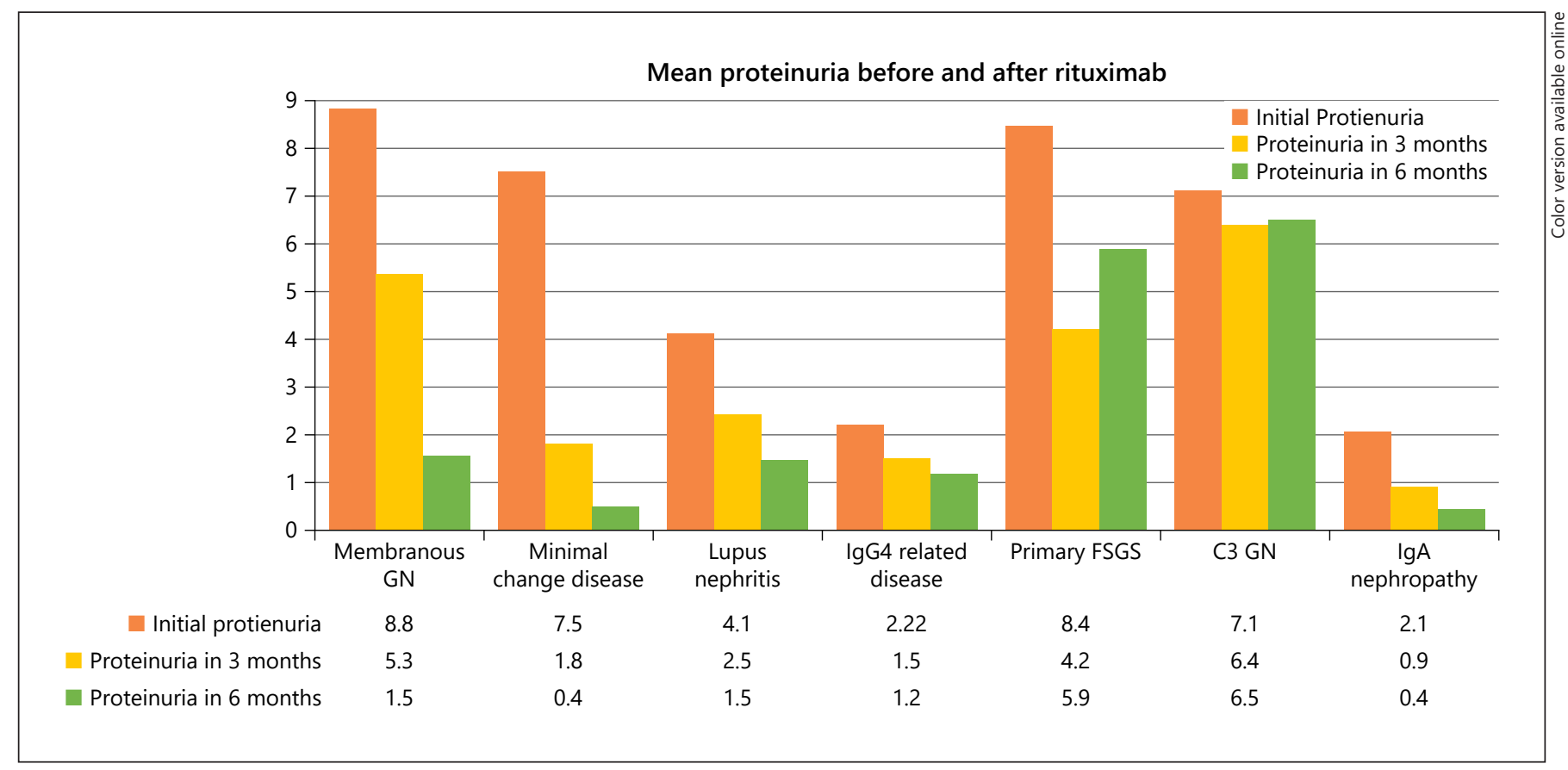

Fig. 2. Mean baseline proteinuria compared with mean proteinuria 3 months and 6 months after RTX administration.

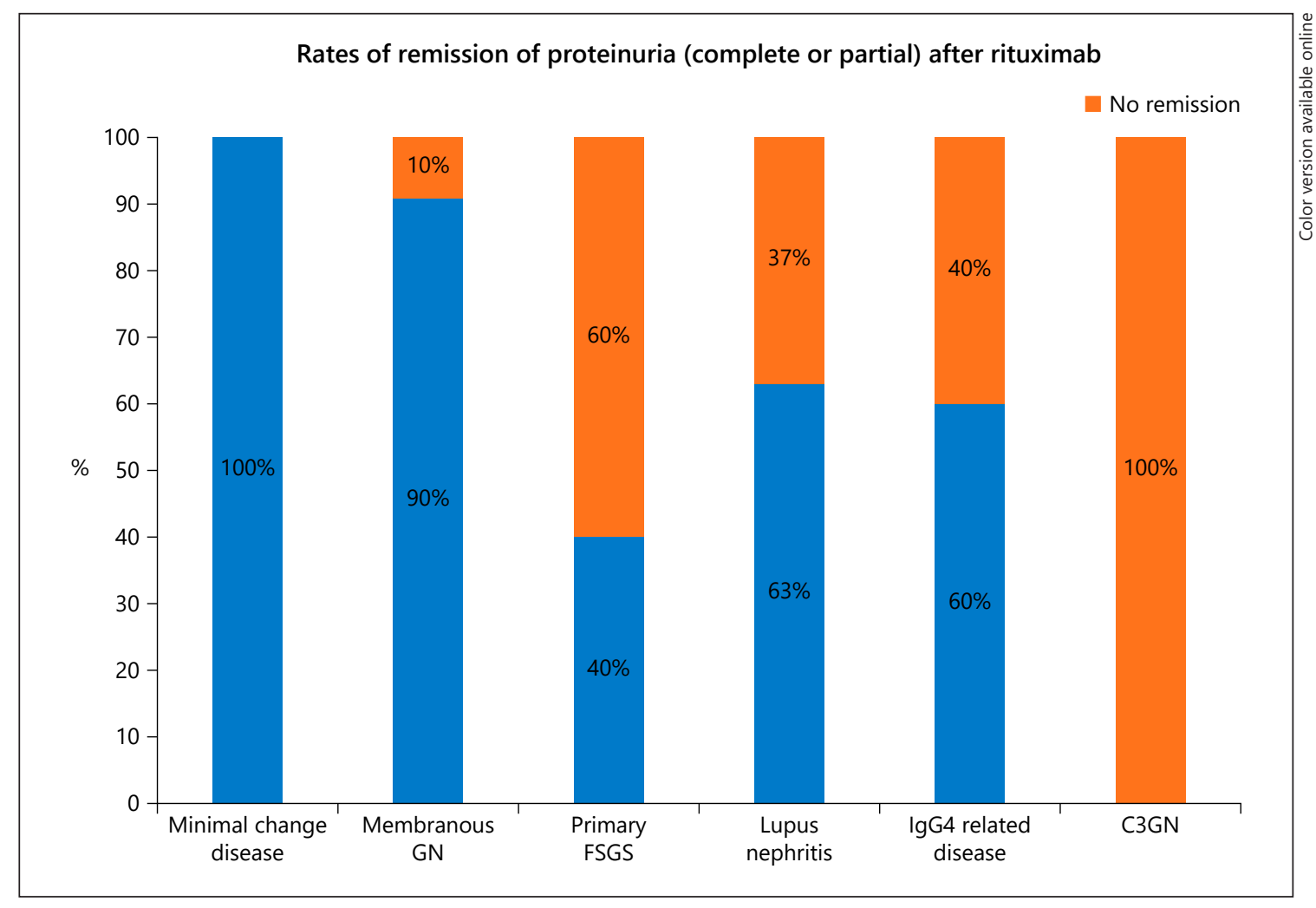

Fig. 3. Rates of remission in each glomerular disease. 
Table 3. Rate of remission between groups supported by the literature (IMN and MCD) and the off-label group (FSGS, LN, C3GN, $\lg \mathrm{AN}$, and IgG4-RD)

\begin{tabular}{lll}
\hline Literature based & $\begin{array}{l}\text { Remission } \\
\text { with RTX }\end{array}$ & $\begin{array}{l}\text { No remission } \\
\text { with RTX }\end{array}$ \\
\hline Literature support, $n(\%)$ & $19(95)$ & $1(5)$ \\
Insufficient data (off-label), $n(\%)$ & $23(56)$ & $18(44)$ \\
\hline
\end{tabular}

$p\left(x^{2}\right.$ test) between remission and no remission $=0.002$.

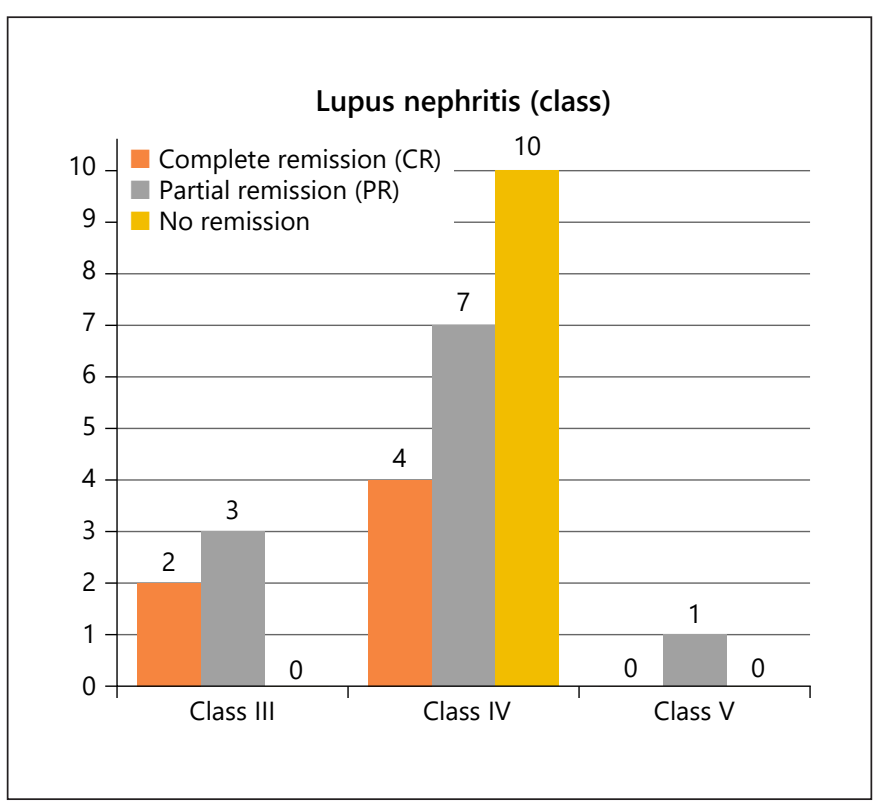

Fig. 4. $C R$ and PR cases in each of the LN classes analyzed.

tial eGFR for $1^{\circ} \mathrm{FSGS}$ patients was $33 \mathrm{~mL} / \mathrm{min}$, which did not improve, and only 2 out of 5 showed PR of proteinuria. The 5 subjects with IgG4-RD who received RTX as salvage therapy showed $60 \%$ remission rate (2 partial, 1 complete). All 5 patients had evidence of tubulointerstitial nephritis, and 4 of 5 patients had received corticosteroids prior to RTX. There was no response in C3GN cases. For IgAN, there was only 1 patient who received RTX in addition to prednisone and mycophenolate mofetil and showed only partial improvement of proteinuria but deterioration in the eGFR. The remission rate was $95 \%$ in diseases with data supporting the RTX group (MCD and $\mathrm{MN})$ versus $56 \%$ in the off-label group Figure $5(p=0.002)$ using the $\chi^{2}$ test (Table 3 ). The crude odds ratio for total remission in the group of diseases supporting RTX use was 14.8 (with confidence interval [CI] 1.81-121). The adjusted odd ratio was 13.1 with ([CI] 1.16-149). The adjusted odds for CR was 2.4 ([CI] $0.50-11.62 p=0.27$ ) and for PR was 1.9 ([CI] 0.52-7.13p=0.32). Meanwhile, the

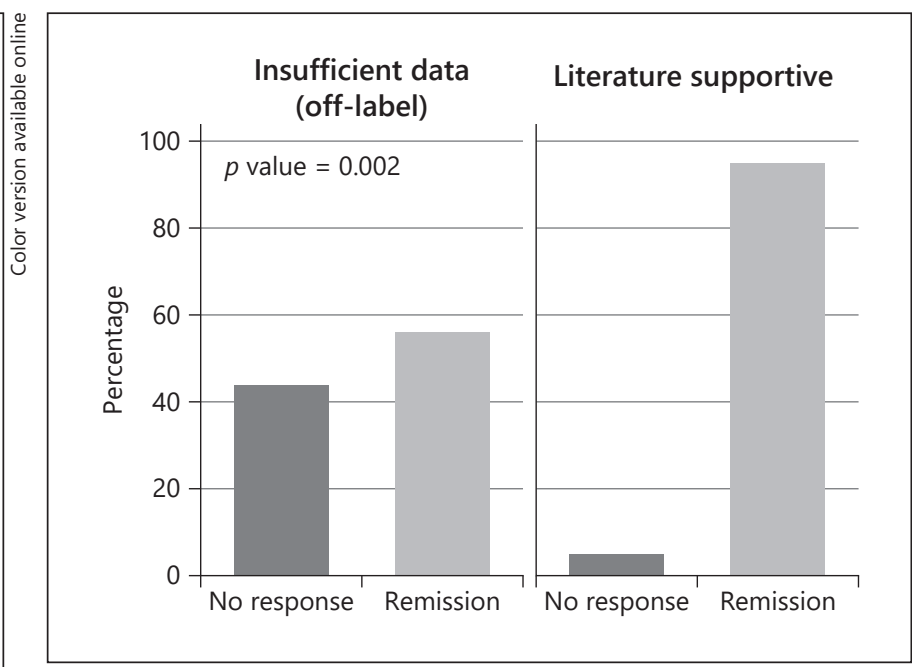

Fig. 5. Rate of remission in the group with literature support (IMN and MCD) versus that of the off-label group (FSGS, LN, C3GN, IgAN, and IgG4-RD).

adjusted odds ratio of total remission for the off-label group was 0.08 ([CI] 0.01-0.86). Furthermore, the odds ratio of CR was $0.38([\mathrm{CI}] 0.11-1.30 p=0.27)$, and odds of PR for the off-label group was $0.51(0.14-1.91 p=0.32)$ (Table 4 ). In the 8 cases of vasculitis, remission was achieved in only 1 pauci-immune vasculitis patients with GFR improving from $36 \mathrm{~mL} / \mathrm{min}$ to $80 \mathrm{~mL} / \mathrm{min}$, with one other patient maintaining an eGFR above $70 \mathrm{~mL} / \mathrm{min}$, and none in the IgA vasculitis patients.

\section{Discussion}

RTX is used in a wide variety of resistant glomerular disease as an addition to corticosteroids and other immunosuppressive medications (Table 1). In some cases, RTX is used as an off-label medication as a last resort to achieve remission by our nephrologists. This study shows that RTX is effective in steroid-dependent and -resistant MCD cases as all of them were on corticosteroid prior to RTX administration. Also, it demonstrates that RTX can be 
Table 4. Crude and adjusted* odds ratio for remission with RTX use based on the literature support versus insufficient data (off-label)

\begin{tabular}{|c|c|c|c|c|c|}
\hline Literature support & Total & $14.8(1.81-121)$ & 0.01 & $13.1(1.16-149)$ & 0.04 \\
\hline $\mathrm{MN}$ and $\mathrm{MCD}$ & Partial & $2.3(0.78-6.98)$ & 0.13 & $1.9(0.52-7.13)$ & 0.32 \\
\hline Insufficient data (off-label) & Total & $0.07(0.01-0.55)$ & 0.01 & $0.08(0.01-0.86)$ & 0.04 \\
\hline FSGS, LN, IgAN, C3GN, and lgG4-RD & Complete & $0.38(0.11-1.30)$ & 0.13 & $0.41(0.86-1.98)$ & 0.27 \\
\hline
\end{tabular}

MN, membranous nephropathy; MCD, minimal change disease; FSGS, focal segmental glomerulosclerosis; LN, lupus nephritis; IgAN, IgA nephropathy; IgG4-RD, IgG4-related disorders; C3GN, C3 glomerulonephritis. * Adjusted for age, gender, initial proteinuria, and initial eGFR.

used to induce remission in MN. This is consistent with the GEMRITUX trial and the recently published MENTOR trial $[4,13]$. Nevertheless, due to the short duration of this study, it was not possible to assess the relapse rate after remission was attained.

According to the Kidney Disease: Improving Global Outcomes Conference Guidelines summary published in 2019, RTX can be considered in refractory cases of LN [14]. The major indication to give RTX was LN. Of the total cohort, there were 27 patients with LN who received RTX. Interestingly, it appears that response to RTX is class-dependent (Table 3). This study included only 1 patient with membranous LN (class V LN) who responded to RTX. This class of LN is caused by deposition of immune complexes in the subepithelial compartment of the glomerular basement membrane that leads to podocyte injury. RTX may be helpful because there is evidence that it promotes podocyte stability [15]. The LUNAR trial [8] did not include any patient with class V LN. A small retrospective study involved 15 patients with pure class $\mathrm{V}$ LN (membranous) showed remission of $87 \%$ with RTX as monotherapy [16]. However, there are no published randomized controlled trials to confirm these findings.

There were only 5 cases of $1^{\circ}$ FSGS; four of these subjects were treated with corticosteroids prior to RTX. Only 2 were able to achieve PR; none of the patients achieved CR; thus, it is not possible to draw any conclusions. Other studies have shown that there is no benefit of RTX in steroid-resistant nephrotic syndrome [17-19].

Although the number of patients with IgG4-RD is small (i.e., 5 cases), the response rate of $60 \%$ seems encouraging and is consistent with previously published studies [20]. In addition, there is evidence that RTX reduces the blood level of IgG4 plasmablasts due to B-cell depletion, which is associated with disease activity [21].
The role of RTX in C3GN has been mentioned in a few case reports but is not clear $[10,22]$. The three C3GN cases in this study failed to respond to RTX. This disease is rare and result from abnormal regulation of the complement pathways [23]. RTX may work better in cases when complement dysregulation is due to $\mathrm{C} 3 \mathrm{NeF}$ or antifactor $\mathrm{H}$ autoantibodies as they seem to be potential treatment targets.

This study included only one subject with IgAN; this patient had a significant decline in the eGFR from $90 \mathrm{~mL} /$ min to $60 \mathrm{~mL} / \mathrm{min}$ despite some reduction in proteinuria. The recent small randomized controlled trial by Lafayette et al. [7] showed that RTX did not significantly improve renal function or proteinuria. RTX is considered standard therapy for induction and maintenance of remission in ANCA-associated vasculitis [24]. However, in our cohort, RTX was an add-on therapy and was not the first choice for induction of remission. This is perhaps partly because cyclophosphamide is still considered the first choice. Given the success of RTX in the treatment of ANCA-associated vasculitis reported in the literature [24], the outcome of our patients with ANCA-associated vasculitis treated with RTX was disappointing.

Even though this study shed light on the clinical use of RTX in Kuwait, it has important limitations that must be highlighted. One limitation is that it is a small and retrospective study, making it more liable to bias and makes it challenging to address all possible confounders. Another limitation is that this study used two different RTX regimens. RTX was used as salvage therapy in addition to previously administered immunosuppressive medications, e.g., corticosteroids, mycophenolate mofetil and CNI. Finally, anti-PLA2R serology was unavailable for the IMN patients, but antibodies were positive in all the biopsies. 
Despite the aforementioned limitations, this study may help in deciding on the optimal use of RTX. It will assist in more conservative use of this medication in glomerular diseases where there is little robust evidence on optimal therapy. At the same time, it may encourage the use of RTX for patients when it is indicated, e.g., in idiopathic MN and steroid-dependent MCD. For LN, the response to RTX might be class-dependent, especially class $\mathrm{V}$. The issue of proper use is important as a RTX dose of $500 \mathrm{mg}$ ( $50 \mathrm{~mL}$ vial) costs roughly 360 Kuwaiti Dinars (approximately USD 1,200). Healthcare coverage in $\mathrm{Ku}$ wait is universal, and RTX is provided free of charge in Ministry of Health hospitals, which makes it readily available to most nephrologists. Further studies are warranted on kidney diseases that are not responsive to RTX, such as complement-mediated C3GN or ANCA-associated vasculitis.

\section{Conclusions}

This study provides insight into the real-world use of RTX for a variety of indications outside of clinical trials. Our data are in line with the literature which supports the use of RTX in resistant MCD and MN, where remission rates are higher. Nevertheless, RTX use in other GN is still not clear despite some success in LN and IgG4$\mathrm{RD}$. Due to the small number of vasculitis patients, we cannot comment on the lack of effectiveness of RTX despite its reported effectiveness. In general, this study provides significant insight on RTX usage for GN in $\mathrm{Ku}$ - wait. It also underscores the need for collaborative studies on large samples sizes to address the need for future RCTs.

\section{Statement of Ethics}

This study was approved by the Ministry of Health - Kuwait University Joint Committee on Medical and Scientific Research.

\section{Conflict of Interest Statement}

The authors declare that they have no competing interests.

\section{Funding Sources}

This work was not funded.

\section{Author Contributions}

All the authors contributed to the design of this study, the acquisition, analysis, interpretation of data, the drafting of the study, and the final approval of this manuscript. All the authors agree to be accountable for all aspects of this study in ensuring that questions related to the accuracy and integrity of any part of the study are appropriately investigated and resolved.

\section{Data Availability Statement}

Data can be provided by the corresponding author in case of a reasonable request.

\section{References}

1 Pescovitz MD. Rituximab, an anti-CD20 monoclonal antibody: history and mechanism of action. Am J Transplant. 2006;6(5 Pt 1):859-66.

2 Stone JH, Merkel PA, Spiera R, Seo P, Langford CA, Hoffman GS, et al. Rituximab versus cyclophosphamide for ANCA-associated vasculitis. N Engl J Med. 2010;363(3):221-32.

3 Fervenza FC, Cosio FG, Erickson SB, Specks U, Herzenberg AM, Dillon JJ, et al. Rituximab treatment of idiopathic membranous nephropathy. Kidney Int. 2008;73(1):117-25.

4 Fervenza FC, Appel GB, Barbour SJ, Rovin BH, Lafayette RA, Aslam N, et al. Rituximab or cyclosporine in the treatment of membranous nephropathy. N Engl J Med. 2019; 381(1):36-46.

5 Iwabuchi Y, Takei T, Moriyama T, Itabashi $\mathrm{M}$, Nitta K. Long-term prognosis of adult patients with steroid-dependent minimal change nephrotic syndrome following rituximab treatment. Medicine. 2014;93(29):e300.

6 Fenoglio R, Sciascia S, Beltrame G, Mesiano P, Ferro M, Quattrocchio G, et al. Rituximab as a front-line therapy for adult-onset minimal change disease with nephrotic syndrome. Oncotarget. 2018;9(48):28799-804.

7 Lafayette RA, Canetta PA, Rovin BH, Appel GB, Novak J, Nath KA, et al. A randomized, controlled trial of rituximab in IgA nephropathy with proteinuria and renal dysfunction. J Am Soc Nephrol. 2017;28(4):1306-13.

8 Rovin BH, Furie R, Latinis K, Looney RJ, Fervenza FC, Sanchez-Guerrero J, et al. Efficacy and safety of rituximab in patients with active proliferative lupus nephritis: the lupus nephritis assessment with rituximab study. Arthritis Rheum. 2012;64(4):1215-26.

9 Cassia M, Alberici F, Gallieni M, Jayne D. Lupus nephritis and B-cell targeting thera- py. Expert Rev Clin Immunol. 2017;13(10): 951-62.

10 Giaime P, Daniel L, Burtey S. Remission of C3 glomerulopathy with rituximab as only immunosuppressive therapy. Clin Nephrol. 2015;83(1):57-60.

11 Laluck BJ Jr, Cattran DC. Prognosis after a complete remission in adult patients with idiopathic membranous nephropathy. Am J Kidney Dis. 1999;33(6):1026-32.

12 Troyanov S, Wall CA, Miller JA, Scholey JW, Cattran DC; Toronto Glomerulonephritis Registry Group. Idiopathic membranous nephropathy: definition and relevance of a partial remission. Kidney Int. 2004;66(3):1199-205.

13 Dahan K, Debiec H, Plaisier E, Cachanado M, Rousseau A, Wakselman L, et al. Rituximab for severe membranous nephropathy: a 6-month trial with extended follow-up. J Am Soc Nephrol. 2017;28(1):348-58. 
14 Rovin BH, Caster DJ, Cattran DC, Gibson KL, Hogan JJ, Moeller MJ, et al. Management and treatment of glomerular diseases (part 2): conclusions from a kidney disease: improving global outcomes (KDIGO) controversies conference. Kidney Int. 2019;95(2):281-95.

15 Yu F, Haas M, Glassock R, Zhao MH. Redefining lupus nephritis: clinical implications of pathophysiologic subtypes. Nat Rev Nephrol. 2017;13(8):483-95.

16 Chavarot N, Verhelst D, Pardon A, Caudwell V, Mercadal L, Sacchi A, et al. Rituximab alone as induction therapy for membranous lupus nephritis: a multicenter retrospective study. Medicine. 2017;96(27):e7429.

17 Ochi A, Takei T, Nakayama K, Iwasaki C, Kamei D, Tsuruta Y, et al. Rituximab treatment for adult patients with focal segmental glomerulosclerosis. Intern Med. 2012;51(7): 759-62.
18 Fernandez-Fresnedo G, Segarra A, Gonzalez E, Alexandru S, Delgado R, Ramos N, et al. Rituximab treatment of adult patients with steroid-resistant focal segmental glomerulosclerosis. Clin J Am Soc Nephrol. 2009;4(8): 1317-23.

19 Kong WY, Swaminathan R, Irish A. Our experience with rituximab therapy for adult-onset primary glomerulonephritis and review of literature. Int Urol Nephrol. 2013;45(3):795802.

20 Ebbo M, Grados A, Samson M, Groh M, Loundou A, Rigolet A, et al. Long-term efficacy and safety of rituximab in IgG4-related disease: data from a French nationwide study of thirty-three patients. PLoS One. 2017; 12(9):e0183844.
21 Wallace ZS, Mattoo H, Carruthers M, Mahajan VS, Della Torre E, Lee H, et al. Plasmablasts as a biomarker for IgG4-related disease, independent of serum IgG4 concentrations. Ann Rheum Dis. 2015;74(1):190-5.

22 Rousset-Rouviere C, Cailliez M, Garaix F, Bruno D, Laurent D, Tsimaratos M. Rituximab fails where eculizumab restores renal function in C3nef-related DDD. Pediatr Nephrol. 2014;29(6):1107-11.

23 Smith RJH, Appel GB, Blom AM, Cook HT, D'Agati VD, Fakhouri F, et al. C3 glomerulopathy - understanding a rare complementdriven renal disease. Nat Rev Nephrol. 2019; 15(3):129-43.

24 Raffray L, Guillevin L. Rituximab treatment of ANCA-associated vasculitis. Expert Opin Biol Ther. 2020 Aug;20(8):899-910. 